\title{
Evaluation of a Brief, Tailored Skin Cancer Risk Assessment and Referral Intervention for Community-Based Health Educators: Brief Report
}

\author{
Julie Williams Merten ${ }^{1}$ (D. Jean Newell ${ }^{1}$
}

Accepted: 11 July 2021 / Published online: 24 July 2021

(c) American Association for Cancer Education 2021

\begin{abstract}
Skin cancer rates are rising and earlier detection through screening leads to better outcomes. Health educators in community centers are well-situated to assess skin cancer risk, deliver tailored health information, and refer high-risk patients to a dermatologist for a screening. This study describes the evaluation of a brief, tailored skin cancer risk assessment, and referral intervention training. The training was developed as part of a pilot feasibility study and seven health educators were trained in an interactive session. Knowledge, attitudes, and efficacy were assessed before and after training. Health educators showed overall improvements of $22 \%$ in skin cancer risk knowledge, $50 \%$ improvement in positive attitudes toward assessment and referral, and a $40 \%$ increase in efficacy to intervene with patients. Community-based health educators that completed the training were able to successfully assess their patients for skin cancer risk and refer high-risk patients for a screening with a healthcare provider.
\end{abstract}

Keywords Skin cancer $\cdot$ Screening $\cdot$ Brief intervention $\cdot$ Health behavior $\cdot$ Evaluation $\cdot$ Training

\section{Introduction}

Screening, brief intervention, and referral to treatment (SBIRT) is a universal, low cost approach to screen a target population using brief, valid, and reliable screening instruments. A health coach can identify the person's level of risk, and provide a quick intervention ranging from information for low-risk individuals to referral for treatment for high-risk individuals [1-4]. SBIRT was originally applied to alcohol use, and then adapted for drug use, and the concept is easily modified for other behaviors [6-8].

We applied the SBIRT approach to skin cancer risk using the brief skin cancer assessment tool (BRAT), an epidemiologically based tool that can be reliably used to assess skin cancer risk in population-based interventions [1]. The instrument demonstrated good reproducibility in several separate studies to classify patients into low-, moderate-,

Julie Williams Merten

JMerten@unf.edu

1 Department of Public Health, Brooks College of Health, University of North Florida, 1 UNF Drive, Jacksonville, FL 32224, USA and high-risk categories. A guide was developed for health coaches to use with patients including a rationale for the importance of skin cancer prevention including UV protection and screening, a script for administering the BRAT, and steps for referring higher risk patients for a total body skin examination.

This study describes the evaluation of a brief, tailored skin cancer risk assessment and referral intervention for community-based health educators.

\section{Methods}

Institutional Research Board (IRB) approval was obtained from the University of North Florida and Baptist Health Research departments. Research and development approval was also obtained from Baptist MD Anderson Cancer Centers. The training program was part of a pilot feasibility study of a brief, tailored skin cancer risk assessment and referral intervention delivered in community-based health education centers in Northeast Florida. 


\section{Training Program}

The training program was developed by a Master Certified Health Education Specialist with guidance from health educators, dermatologists, primary care providers, and skin cancer researchers. Focus groups were conducted with community-based health educators to customize the training program. Prior to the training, participants completed a pre-test assessment of knowledge, attitudes, and confidence. The training was delivered in a 2-h session with a module to improve skin cancer knowledge that included an overview of skin cancer rates, risk factors, screening and treatment options, and current clinical practice guidelines.

Barriers to total body skin examinations were discussed and explored. The lead facilitator discussed how to address these concerns with patients. Inclusion criteria for the intervention were discussed. Health educators were provided with a sample script to use to invite patients to participate and provide informed consent. Then they were introduced to the brief skin cancer assessment tool (BRAT), the three levels of risk, and scoring guide, which tailored intervention to use depending on score.

Each level of skin cancer risk was discussed along with the appropriate tailored educational intervention. Patients that score low risk on the BRAT are to be notified of their score by the health educator, the meaning of the score, and provided literature on skin cancer prevention including sun protection and resources on skin cancer screenings to share with friends and family. For patients that screen at moderate risk, the health educator practiced providing feedback on the score and conducts a brief intervention to promote sun protection, show the participant how to use the American Academy of Dermatology DETECT Skin Cancer: Body Mole Map that encourages skin cancer selfexaminations, and provide information on total body skin examinations conducted by a healthcare provider. This script takes about $10 \mathrm{~min}$ and uses a motivational interviewing strategy to assess readiness to change, establish goals, and plan strategies for change. For the patients that score in the high-risk category, the health educator practiced providing feedback on the score and a brief intervention to refer them to a healthcare provider for a total body skin examination. The health educator was coached to provide the patient with a list of local dermatologists and free skin cancer screening resources. This process takes about 10 to $15 \mathrm{~min}$ and also uses a motivational interviewing strategy. Health coaches will also establish a plan for follow-up to assess if a patient followed referral recommendations.

After the knowledge module and introduction of the risk assessment and intervention, health educators were provided a Frequently Asked Questions list of common skin cancer questions with short bulleted answers along with the training manual, QR code link to the consent and assessment, risk factor scorecards for patients, and educational materials to use with their patients. Health educators completed a post-test assessment of knowledge, attitude, and efficacy via electronic survey 1 week after the training.

\section{Program Evaluation}

Prior to the training session, health educators completed a survey to establish their baseline knowledge and attitudes and confidence in delivering skin cancer prevention education to patients. Further, the health educators' attitudes toward skin cancer risk assessment and intervention were assessed with five five-point Likert scale questions with a scale ranging from one (strongly disagree) to five (strongly agree). Finally, the health educators' confidence in providing skin cancer risk assessment and tailored education were assessed with a series of four questions with five-point Likert scale questions with a scale ranging from one (not very confident) to five (very confident).

The same eighteen questions assessing knowledge, attitudes, and confidence were administered 1 week after the training along with questions about the effectiveness of the training and suggestions for improvement. Answers were linked but anonymous to protect health educator privacy.

\section{Fidelity Testing}

Health educators started the intervention 1 week after the training. Researchers provided a fidelity checklist for participants to ensure the intervention was consistently delivered. The checklist included all required intervention steps and approximate time it took to deliver the intervention.

\section{Analysis}

Data was analyzed using SPSS version 26 using simple descriptive statistics to analyze baseline data. The cumulative scores for each training category including knowledge, efficacy, and attitudes were calculated in aggregate. Each participant's score was analyzed using Wilcoxon matchedpairs signed ranks test pre- and post-training.

\section{Results}

\section{Participant Characteristics}

Seven community-based health educators attended the training session via Webex. We used Webex due to COVID-19 precautions. Seventy-one percent of the participants $(n=5)$ 
were female with a mean age of $30.2(\mathrm{SD}=8.91)$. Three of the health educators were African-American, one was Asian, Pacific-Islander, one was Hispanic, and two were Caucasian. All participants had degrees in a health-related field including public health $(n=5)$, nursing $(n=1)$, and allied health $(n=1)$. There was a mix of recent graduates and seasoned professional health educators. The participants reported they had not attended any prior skin cancer screening training.

\section{Training Variables}

The participating health educators reported significant improvements in the three training domains. Knowledge pretest scores ranged from 42.9 to $100 \%$ but improved to $100 \%$ for all questions after the intervention. There was a $50 \%$ overall change in positive attitudes toward asking and advising their clients about skin cancer, using the skin cancer risk assessment instrument, and referring patients to a dermatologist for TBSE. And overall efficacy scores increased by $40 \%$ with health educators reporting significantly more confidence in using the skin cancer assessment tool to determine risk and refer individuals at increased risk to a dermatologist for a TBSE. The complete attitudes and efficacy scores are reported in Table 1.

\section{Training Feedback and Fidelity}

The seven health educator participants reported (agreed or strongly agreed) the training was comprehensive, objectives were clearly defined, the resources were valuable, and they felt prepared to deliver the intervention to their patients. They rated the training materials as very good on a fivepoint scale ranging from very poor to very good.

Intervention fidelity was randomly assessed in 50\% $(n=81)$ of the patients with the intervention length ranging from 4 to more than $25 \mathrm{~min}$. The health educators successfully completed all steps of the intervention.

\section{Discussion}

The training for health educators to deliver a brief, tailored skin cancer risk assessment and referral intervention showed improvements in knowledge, attitudes, and efficacy. After the training, the health educators showed marked confidence improvements in using the assessment tool to determine their patients' skin cancer risk and their ability to refer those patients for a total body skin examination by a dermatologist. For comparison, we were unable to locate any other training evaluations for brief, tailored skin cancer risk assessments given the novel approach of this intervention. The evaluation findings of the training program are promising and the health educators successfully delivered the intervention to reach the desired study participation goals even during COVID-19 limitations.

As with any study, there are limitations with this evaluation. Our team of health educators is relatively small so it limits our ability to generalize the findings. Further, the study was limited to health educators, which is a population that will likely have a higher level of knowledge about skin cancer risks, better attitudes about the importance of skin cancer screening, and increased efficacy in their ability to effectively counsel their patients. The training may need to be adjusted when the intervention is expanded to other providers such as personal trainers, nail technicians, hairdressers, cosmetologists, and swim instructors who may not have a background in health. Additionally, the study was limited to Northeast Florida which is an area that may have a higher acuity of skin cancer given the location situated in the self-proclaimed "Sunshine State."

Table 1 Attitudes and efficacy assessment before and after training

\begin{tabular}{|c|c|c|c|c|}
\hline \multirow[b]{2}{*}{ I always ask about skin cancer during a health coaching session } & \multirow{2}{*}{$\begin{array}{l}\text { Pre-test score } \\
\text { Median (IQR) } \\
1(1,3)\end{array}$} & \multirow{2}{*}{$\begin{array}{l}\text { Post-test score } \\
\text { Median (IQR) } \\
5(4,5)\end{array}$} & \multicolumn{2}{|c|}{$\begin{array}{l}\text { Paired } \\
\text { differences } \\
\text { Median (IQR) } P\end{array}$} \\
\hline & & & $3(3,3)$ & 0.0005 \\
\hline $\begin{array}{l}\text { I feel it is part of my job as a health professional to be able to identify individuals at } \\
\text { increased risk for skin cancer and refer them }\end{array}$ & $5(5,5)$ & $5(5,5)$ & & \\
\hline I feel I can appropriately advise my clients about sun safety and its effect on skin cancer & $2(1,3)$ & $5(5,5)$ & $2(1,3)$ & 0.0005 \\
\hline I know how to assess a person's skin cancer risk using the BRAT assessment & $1(1,3)$ & $5(5,5)$ & $3(3,3)$ & 0.0005 \\
\hline I can define the characteristics of someone at increased risk for skin cancer & $2(1,3)$ & $5(4,5)$ & $2(1,3)$ & 0.0013 \\
\hline \multicolumn{5}{|l|}{ How confident do you feel to } \\
\hline Describe impact of UV exposure on skin cancer risk & $3(3,4)$ & $5(5,5)$ & $2(1,3)$ & 0.0005 \\
\hline Provide brief advice on skin cancer to your patients & $3(2,4)$ & $5(5,5)$ & $2(1,3)$ & 0.001 \\
\hline Use assessment tool to determine skin cancer risk & $1(1,2)$ & $5(5,5)$ & $3(3,3)$ & 0.0005 \\
\hline Refer individuals at increased risk for skin cancer & $2(1,3)$ & $5(5,5)$ & $2(1,3)$ & 0.0006 \\
\hline
\end{tabular}

Scale for attitudes ( 1 to $5 ; 1$ strongly disagree, 5 strongly agree), scale for efficacy ( 1 to $5 ; 1$ not very confident, 5 very confident) 
Given the limitations, the evaluation revealed the training was comprehensive, high-quality, and effective. Further, we solicited and received excellent feedback from the health educators on how to improve and tailor the training for future partners.

\section{Conclusion}

This evaluation showed that health educators can be trained to effectively deliver a brief, tailored skin cancer risk assessment and referral intervention. Future studies will examine the training effectiveness with other providers including personal trainers, nail technicians, hairdressers, cosmetologists, and swim instructors who have ongoing relationships with people and the opportunity to discuss skin cancer risk.

Author Contribution JM contributed to the study conception and design. Material preparation, data collection, and analysis were performed by JM and JN. The first draft of the manuscript was written by JM and all authors commented on previous versions of the manuscript. All authors read and approved the final manuscript.

Funding The study was funded by the University of North Florida Brooks College of Health Brooks Professorship research grant.

\section{Declarations}

Ethics Approval This study was approved by the UNF IRB Office.

Consent to Participate Informed consent was obtained from all individual participants included in the study.

\section{Consent for Publication Not applicable.}

Conflict of Interest The authors have no conflicts of interest to declare that are relevant to the content of this article. All authors certify that they have no affiliations with or involvement in any organization or entity with any financial interest or non-financial interest in the subject matter or materials discussed in this manuscript.
Financial Interests The authors have no relevant financial or non-financial interests to disclose. The authors have no financial or proprietary interests in any material discussed in this article.

\section{References}

1. Kirsner RS, Muhkerjee S, Federman DG (1999) Skin cancer screening in primary care: prevalence and barriers. J Am Acad Dermatol 41(4):564-566

2. Glanz K, Schoenfeld E, Weinstock MA, Layi G, Kidd J, Shigaki DM (2003) Development and reliability of a brief skin cancer risk assessment tool. Cancer Detect Prev 27:311-315

3. National Institute on Alcohol Abuse and Alcoholism. Screening and brief intervention, part 1-an overview. Alcohol Research and Health. 2004/2005; 28(1). Available at: http://pubs.niaaa.nih.gov/ publications/arh28-1/toc28-1.htm.

4. National Institute on Alcohol Abuse and Alcoholism. Screening and brief intervention, part II-a focus on specific settings. Alcohol Research and Health. 2004/2005; 28(2). Available at: http://pubs. niaaa.nih.gov/publications/arh28-2/toc28-2.htm.

5. US Preventive Services Task Force (2004) Screening and behavioral counseling interventions in primary care to reduce alcohol misuse: recommendation statement. Ann Intern Med 140(7):554-556

6. Madras BK, Compton WM, Avula D, Stegbauer T, Stein JB, Clark HW (2009) Screening, brief interventions, referral to treatment (SBIRT) for illicit drug and alcohol use at multiple healthcare sites: comparison at intake and 6 months later. Drug Alcohol Depend 99(1-3):280-295

7. Kaner E, Dickinson HO, Beyer F, Pienaar E, Schlesinger C, Campbell F, Saunders JB, Burnand B, Heather N (2009) The effectiveness of brief alcohol interventions in primary care settings: a systematic review. Drug Alcohol Rev 28(3):301-323

8. Humeniuk R, Ali R, Babor T, Souza-Formigoni ML, Boerngen de Lacerda R, Ling W, McRee B, Newcombe D, Pal H, Poznyak V, Simon S, Vendetti J (2012) A randomized controlled trial of a brief intervention for illicit drugs linked to the Alcohol, Smoking and Substance Involvement Screening Test (ASSIST) in clients recruited from primary health-care settings in four countries. Addiction 107(5):957-966

Publisher's Note Springer Nature remains neutral with regard to jurisdictional claims in published maps and institutional affiliations. 